\title{
Valproic acid inhibits adhesion of vincristine- and cisplatin-resistant neuroblastoma tumour cells to endothelium
}

\author{
RA Blaheta', M Michaelis², I Natsheh', C Hasenberg', E Weich', B Relja', D Jonas', HW Doerr² and J Cinatl Jr,2 \\ 'Zentrum der Chirurgie, Klinik für Urologie und Kinderurologie, Johann Wolfgang Goethe-Universität, Frankfurt am Main, Germany; ${ }^{2}$ Zentrum der \\ Hygiene, Institut für Medizinische Virologie, Johann Wolfgang Goethe-Universität, 60590 Frankfurt am Main, Germany
}

\begin{abstract}
Drug resistance to chemotherapy is often associated with increased malignancy in neuroblastoma (NB). In pursuit of alternative treatments for chemoresistant tumour cells, we tested the response of multidrug-resistant SKNSH and of vincristine (VCR)-, doxorubicin (DOX)-, or cisplatin (CDDP)-resistant UKF-NB-2, UKF-NB-3 or UKF-NB-6 NB tumour cell lines to valproic acid (VPA), a differentiation inducer currently in clinical trials. Drug resistance caused elevated NB adhesion (UKF-NB-2 ${ }^{\mathrm{VCR}}$, UKF-NB-2 ${ }^{\mathrm{DOX}}$,

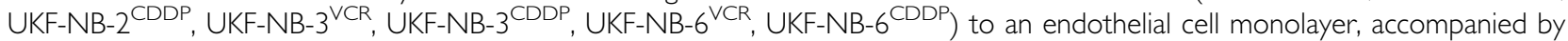
downregulation of the adhesion receptor neural cell adhesion molecule (NCAM). Based on the UKF-NB-3 model, N-myc proteins were enhanced in UKF-NB-3 $3^{\mathrm{VCR}}$ and UKF-NB-3 ${ }^{\mathrm{CDDP}}$, compared to the drug naïve controls. p73 was diminished, whereas the p73 isoform deltaNp73 was upregulated in UKF-NB-3 $3^{\mathrm{VCR}}$ and UKF-NB-3 ${ }^{\mathrm{CDDP}}$. Valproic acid blocked adhesion of UKF-NB-3 $3^{\mathrm{VCR}}$ and UKFNB-3 $3^{C D D P}$, but not of UKF-NB-3 ${ }^{\mathrm{DOX}}$, and induced the upregulation of NCAM surface expression, NCAM protein content and NCAM coding mRNA. Valproic acid diminished N-myc and enhanced p73 protein level, coupled with downregulation of deltaNp73 in UKF-NB-3 ${ }^{\text {VCR }}$ and UKF-NB-3 ${ }^{C D D P}$. Valproic acid also reverted enhanced adhesion properties of drug-resistant UKF-NB-2, UKFNB-6 and SKNSH cells, and therefore may provide an alternative approach to the treatment of drug-resistant NB by blocking invasive processes.
\end{abstract}

British Journal of Cancer (2007) 96, I699-1706. doi:I0.I038/sj.bjc.6603777 www.bjcancer.com

Published online 15 May 2007

(C) 2007 Cancer Research UK

Keywords: neuroblastoma; chemoresistance; valproic acid; adhesion; NCAM

Multiple-agent chemotherapy is the conventional therapy for patients with advanced stages of neuroblastoma (NB) and disseminated NB. However, drug resistance arises in the majority of stage IV and relapsed NB, often leading to treatment failure (Keshelava et al, 1998). Development of novel antitumoural strategies is therefore highly desired to overcome resistance mechanisms and to prevent tumour progression. Molecules modulating cellular function have been identified in the majority of tumours and their manipulation might be the key to decreasing malignancy.

Histone deacetylases (HDAC) represent one of the most important intracellular targets, as these molecules modulate a wide variety of cellular functions. Abnormal histone acetylation status can result in undesirable phenotypic changes, including developmental disorders and cancer. Indeed, aberrant histone acetylation may be an aetiological factor in several types of cancer by derepressing gene transcription. Hence, HDAC inhibitors may be useful for cancer prevention, due to their ability to 'reactivate' the expression of epigenetically silenced genes, including those involved in differentiation, invasion and metastasis. Most notably, recent data indicate that HDAC inhibition may be successful in treating refractory or relapsing tumours after conventional chemotherapy. Histone deacetylase inhibition has been demon-

\footnotetext{
* Correspondence: Professor Dr J Cinatl Jr;

E-mail: cinat|@em.uni-frankfurt.de

Received 10 January 2007; revised 4 April 2007; accepted 12 April 2007; published online I5 May 2007
}

strated to block cell growth of drug-resistant small-cell lung cancer lines (Tsurutani et al, 2003), abrogate resistance in breast cancer cells (Hirokawa et al, 2005) and induce apoptosis in drug-resistant ovarian cancer cells (Sonnemann et al, 2006), myeloma cells (Maiso et al, 2006) and hepatoma cell lines (Pathil et al, 2006).

The branched-chain fatty acid valproic acid (VPA) has been shown to possess HDAC inhibitory properties and to affect the growth and survival of tumour cells in vitro and in vivo (Cinatl et al, 1996; Blaheta et al, 2005). This is highly relevant since VPA is an established drug in the long-term therapy of epilepsy. It can be applied orally, negative side effects are rare and it demonstrates expedient pharmacokinetic properties. To clearly assess whether VPA might be of benefit in treating relapsed NB, we evaluated the potential of therapeutic VPA concentration to block the interaction of drug-resistant NB cells with vascular endothelium. The experiments were based on an in vitro model of acquired drug resistance (Kotchetkov et al, 2003, 2005), closely resembling progressive NB disease through long-term treatment of $\mathrm{NB}$ cell lines with vincristine (VCR), cisplatin (CDDP) or doxorubicin (DOX) to establish the resistant tumour cell sublines UKF-NB-3 ${ }^{\mathrm{VCR}}$, UKF-NB-3 ${ }^{\mathrm{CDDP}}$ and UKF-NB-3 ${ }^{\text {DOX }}$. A co-culture binding assay allowed the analysis of NB cells that adhered to an endothelial cell monolayer. Since surface receptors are strongly involved in tumour invasion and data have indicated that changes in neural cell adhesion molecule (NCAM, CD56) expression play an essential part in the progression of NB, we investigated NCAM expression (Blaheta et al, 2002), and the NCAM regulating proteins $\mathrm{N}$-myc, p73 and deltaNp73 (Blaheta et al, 2004) under the influence of VPA. 


\section{MATERIALS AND METHODS}

\section{Cell cultures and induction of drug resistance}

The N-myc amplified human NB cell lines UKF-NB-2, UKF-NB-3 and UKF-NB-6 were established in our laboratory from bone marrow metastases. The CDDP-, VCR- and DOX-resistant UKFNB-2, UKF-NB-3 and UKF-NB-6 sublines were established by exposing the parental cells to increasing concentrations of the drugs. Solutions of CDDP (Gry-Pharma, Kirchzarten, Germany), VCR (Sigma-Aldrich, Deisenhofen, Germany) and DOX (Farmitalia, Milan, Italy) were prepared in accordance to the manufacturer's instructions. The initial CDDP, VCR and DOX concentrations were 50, 0.2 and $2 \mathrm{ng} \mathrm{ml}^{-1}$ medium, respectively. The resistant sublines were grown for more than 6 months in Iscove's modified Dulbecco's medium (Gibco, Karlsruhe, Germany) supplemented with $10 \%$ fetal calf serum (FCS, Gibco) and either $10 \mathrm{ng} \mathrm{ml}^{-1}$ VCR (UKF-NB-2 $\mathrm{VCR}$, UKF-NB-3 ${ }^{\mathrm{VCR}}$, UKF-NB-6 ${ }^{\mathrm{VCR}}$ ), $20 \mathrm{ng} \mathrm{ml}^{-1}$ DOX (UKF-NB-2 ${ }^{\text {DOX }}$, UKF-NB-3 ${ }^{\text {DOX }}$, UKF-NB- $6^{\text {DOX }}$ ), or $1000 \mathrm{ng} \mathrm{ml}^{-1}$ CDDP (UKF-NB-2 ${ }^{\mathrm{CDDP}}$, UKF-NB-3 ${ }^{\mathrm{CDDP}}$, UKFNB- $\left.6^{\mathrm{CBDP}}\right)$. The clinical relevance of drug-resistant UKF-NB-2 and UKF-NB-3 sublines has been examined before in a xenograft setting (Kotchetkov et al, 2003, 2005). Multidrug resistant, not N-myc amplified SKNSH, were derived from LGC Promochem, Wesel, Germany. Cells were subcultured at 5-day intervals.

Chemoresistance of the indicated cell lines was verified by the MTT test (Kotchetkov et al, 2003). The resistant cell lines showed at least 20-fold increase in resistance to the drugs, expressed as $\mathrm{IC}_{50}$.

Human endothelial cells (HUVEC) were isolated from human umbilical veins and harvested by enzymatic treatment with chymotrypsin. Human endothelial cells were grown in Medium 199 (M199; Biozol, Munich, Germany), supplemented with 10\% FCS, $10 \%$ pooled human serum, $20 \mu \mathrm{g} \mathrm{ml}^{-1}$ endothelial cell growth factor (Boehringer, Mannheim, Germany), $0.1 \%$ heparin, $100 \mathrm{ng} \mathrm{ml}^{-1}$ gentamycin and $20 \mathrm{~mm}$ HEPES buffer ( $\mathrm{pH} 7.4$ ). Subcultures from passages 2-6 were selected for experimental use.

\section{VPA treatment}

Parental NB tumour cells and their drug-resistant sublines were treated with VPA (gift from GL Pharma GmbH, Lannach, Austria) at a final concentration of $1 \mathrm{~mm}$ for 3 or 5 days. Tumour cell adhesion, NCAM, p73, deltaNp73 and N-myc expression were then measured in VPA-treated cells. Results were compared to untreated controls. Viability of tumour cells in presence of VPA was assessed by propidium iodide dsDNA intercalation.

\section{Tumour cell adhesion}

Human endothelial cells were transferred to six-well multiplates (Falcon Primaria; Becton Dickinson, Heidelberg, Germany) in complete HUVEC medium. When confluency was reached, $0.5 \times 10^{6}$ parental NB tumour cells or their drug-resistant sublines (VPA treated $v s$ non-treated) were carefully added to the HUVEC monolayer for $60 \mathrm{~min}$. Subsequently, non-adherent tumour cells were washed off using warmed $\left(37^{\circ} \mathrm{C}\right) \mathrm{M} 199$. The adherent cells were fixed with $1 \%$ glutaraldehyde and counted in five different fields $\left(5 \times 0.25 \mathrm{~mm}^{2}\right)$ using a phase contrast microscope $(20 \times$ objective $)$ to calculate the mean cellular adhesion rate.

\section{Cell proliferation}

Proliferative activity of NB tumour cells and HUVEC was estimated by the PicoGreen assay as described elsewhere (Blaheta et al, 1998). Briefly, at several time points after plating the cells in six-well multiplates, culture medium was removed and cells were digested with papain $\left(0.125 \mathrm{mg}\right.$ protein $\left.\mathrm{ml}^{-1}\right)$ for $20 \mathrm{~h}$ at $60^{\circ} \mathrm{C}$. Thereafter, the fluorescent dye PicoGreen (MoBiTec, Goettingen,
Germany), which shows high specificity for dsDNA, was added (1:200 dilution) for $10 \mathrm{~min}$ at $20^{\circ} \mathrm{C}$. Fluorescence intensity was determined using a computer-controlled fluorescence reader (Cytofluor 2300 plate scanner; Millipore, Eschborn, Germany) at $\lambda \mathrm{ex}=485 \mathrm{~nm}$ and $\lambda \mathrm{em}=530 \mathrm{~nm}$.

\section{Evaluation of NCAM surface expression}

Neuroblastoma cells were disaggregated mechanically, washed in blocking solution (PBS, $0.5 \% \mathrm{BSA}$ ) and then incubated for $60 \mathrm{~min}$ at $4{ }^{\circ} \mathrm{C}$ with an FITC-conjugated monoclonal antibody anti-CD56 which detects the NCAM 120, 140 and $180 \mathrm{kDa}$ isoform (clone 16.2). Neural cell adhesion molecule expression of NB cells was then measured using a FACscan (Becton Dickinson; FL-1 H (log) channel histogram analysis; $1 \times 10^{4}$ cells/scan) and expressed as mean fluorescence units (MFU). A mouse IgG1-FITC was used as the isotype control.

To explore NCAM localisation, tumour cells were transferred to round cover slips, which were placed in a 24-well multiplate. Upon reaching confluency, cell cultures were washed two times with PBS (with $\mathrm{Ca}^{2+}$ and $\mathrm{Mg}^{2+}$ ) and then fixed in cold $\left(-20^{\circ} \mathrm{C}\right)$ methanol/ acetone $(60 / 40 \mathrm{v} / \mathrm{v})$. Subsequently, cells were washed again with PBS (without $\mathrm{Ca}^{2+}$ and $\mathrm{Mg}^{2+}$ ), and later once with blocking buffer $\left(0.5 \%\right.$ BSA in PBS without $\mathrm{Ca}^{2+}$ and $\left.\mathrm{Mg}^{2+}\right)$. After removing the washing buffer, cells were incubated for $60 \mathrm{~min}$ with FITCconjugated anti-NCAM monoclonal antibody. To prevent photobleaching of the fluorescent dye, cover glasses with stained cells were taken out of the wells and the residual liquid was removed. The cells were then embedded in an antifade reagent/mounting medium mixture (ProLong ${ }^{\mathrm{TM}}$ Antifade Kit, MoBiTec) and mounted on slides. The slides were viewed using a confocal laser-scanning microscope (LSM 10; Zeiss, Jena, Germany) with a plan-neofluar $\times 100 / 1.3$ oil immersion objective.

\section{Western blot analysis}

Total NCAM content in NB cells was evaluated by Western blot analysis: tumour cell lysates were applied to a $7 \%$ polyacrylamide gel and electrophoresed for $90 \mathrm{~min}$ at $60 \mathrm{~V}$. The protein was then transferred to nitrocellulose membranes. After blocking, the membranes were incubated overnight with the anti-NCAM antibody (dilution $1: 1000$ ). HRP-conjugated goat anti-mouse IgG (Upstate Biotechnology, Lake Placid, NY, USA; dilution 1:5000) served as the secondary antibody. The membrane was briefly incubated with ECL detection reagent $\left(\mathrm{ECL}^{\mathrm{TM}}\right.$, Amersham/GE Healthcare, München, Germany) to visualise the proteins and exposed to an x-ray film (Hyperfilm ${ }^{\mathrm{TM}} \mathrm{EC}^{\mathrm{TM}}$, Amersham).

\section{Semi-quantitative reverse transcription/polymerase chain reaction}

Total RNA was extracted and purified with Trizol reagent according to the manufacturer's instructions and treated with RNase-free DNase. The NCAM primer sequences were as follows: for NCAM-180: 5'CGAGGCTGCCTCCGTCAGCACC $3^{\prime}$ and 5'CCGG ATCCATCATGCTTTGCTCTCG $3^{\prime}$; for NCAM-140: $5^{\prime}$ GAACCTG ATCAAGCAGGATGACGG $3^{\prime}$ and $5^{\prime}$ CCGGATCCATCATGCTTTGC TCTCG 3' (Kleinschmidt-DeMasters et al, 1999). Internal controls for the reverse transcription/polymerase chain reaction (RT-PCR) reaction was performed by running parallel reaction mixtures with the housekeeping gene GAPDH: 5'ATCTTCCAGGAGCGAGATCC $3^{\prime}$ and $5^{\prime}$ ACCACTGACACGTTGGCAGT $3^{\prime}$. Ribonucleic acid $(1-10 \mu \mathrm{g})$ was reverse transcribed and the resulting cDNA directly added to the PCR. Amplification reactions $(20 \mu \mathrm{l})$ were performed in the presence of $1 / 10(2 \mu \mathrm{l})$ of the cDNA reaction, with an initial incubation step at $94^{\circ} \mathrm{C}$ for $1 \mathrm{~min}$. Cycling conditions consisted of denaturation at $94^{\circ} \mathrm{C}$ for $1 \mathrm{~min}$, annealing at $55^{\circ} \mathrm{C}$ for $1 \mathrm{~min}$ and extension at $72^{\circ} \mathrm{C}$ for $1 \mathrm{~min}$ over a total of 30 cycles. The reaction 
was completed by another incubation step at $72^{\circ} \mathrm{C}$ for $10 \mathrm{~min}$. The PCR products were subjected to electrophoresis in $2 \%$ agarose gel and visualised by ethidium bromide.

\section{Evaluation of p73, deltaNp73 and N-myc}

$\mathrm{N}$-myc, p73 and deltaNp73 were evaluated by flow cytometry. To allow intracellular staining, tumour cells were fixed and permeabilised by methanol-acetone $\left(1: 1,-20^{\circ} \mathrm{C}\right)$ before the antibodies were added. Monoclonal anti-N-myc antibody was from Calbiochem (clone NCM II 100; mouse IgG1; Calbiochem, Bad Soden, Germany). p73 was measured using monoclonal anti-p73 (clone ER-15, Becton Dickinson). To identify deltaNp73, the monoclonal antibody anti-deltaNp73 (clone 38C674) was purchased from Active Motif (Rixensart, Belgium). Primary antibodies were labelled with goat anti-mouse IgG-FITC. To evaluate background staining, goat anti-mouse IgG-FITC was used.

$\mathrm{N}$-myc was also explored by Western blot analysis using monoclonal antibodies against $\mathrm{N}-\mathrm{myc}(1: 250$, clone NCM II 100 ; mouse IgG1). $\beta$-Actin $(1: 1.000$, mouse; Sigma, Taufkirchen, Germany) served as the internal control.

To investigate $\mathrm{N}$-myc coding mRNA, RT/PCR has been carried out as described above. The $\mathrm{N}$-myc primer sequences were as follows: forward: $5^{\prime}$ GACCACAAGGCCCTCAGTAC $3^{\prime}$; reverse: 5'GTGGATGGGAAGGCATCGTT $3^{\prime}$.

\section{Statistics}

All experiments were performed 3-6 times. Statistical significance was investigated by the Wilcoxon-Mann - Whitney $U$-test. Differences were considered statistically significant at $P<0.05$.

\section{RESULTS}

\section{VPA downregulates cell adhesion of CDDP- and VCR-} resistant UKF-NB-3 tumour cells

Adhesion of UKF-NB-3, UKF-NB-3 $3^{\mathrm{CDDP}}$, UKF-NB-3 ${ }^{\mathrm{VCR}}$ or UKF$\mathrm{NB}-3^{\mathrm{DOX}}$ was quantified $60 \mathrm{~min}$ after plating the cells on to an endothelial cell monolayer (Figure 1). Nearly 200 parental (drugsensitive) UKF-NB-3 cells mm ${ }^{-2}$ were attached to HUVEC during this time $\left(\mathrm{SD}_{\text {interassay }}<50 \%, \mathrm{SD}_{\text {intraassay }}<10 \%\right)$. The amount of adherent cells increased fourfold when UKF-NB-3 became resistant to CDDP or VCR. Doxorubicin resistance did not induce any effects on tumour cell binding to HUVEC. Table 1 provides a profile of cross-resistance among the sublines examined.

The application of $1 \mathrm{mM}$ VPA to UKF-NB-3 $3^{\mathrm{CDDP}}$ or UKF-NB$3^{\mathrm{VCR}}$ significantly blocked the cellular adhesion process (Figure 1). A 5-day incubation period evoked stronger effects than a 3-day incubation period. Notably, treatment of UKF-NB-3 ${ }^{\mathrm{CDDP}}$ with VPA completely reverted the elevated adhesion behaviour induced by drug resistance. Valproic acid also acted on the parental cell lines, as evidenced by a significant downregulation of the number of adherent UKF-NB-3. The 60 -min adhesion rate was reduced by $59.4 \pm 18.6 \%(n=6)$.

The PicoGreen assay did not reveal any proliferative activity during the experiment, which rules out the possibility that adhesion differences between drug resistant, VPA-treated and control NB cells may be caused by different cell growth capacity.

\section{VPA upregulates NCAM surface expression}

Figure 2 depicts one representative histogram analysis of NCAM receptor expression. The histogram presentation concentrates on isotype controls and NCAM-specific fluorescence of untreated $v s$ VPA-treated (5-day treatment)-resistant tumour cells. Results of parental UKF-NB-3 and of resistant tumour cells treated for 3 days with VPA were not inserted, because the histograms overlapped, making the figures unclear. However, the complete experimental data set (MFU \pm s.d.; $n=6$ ) is given below the histogram.

Table I Level of drug resistance, indicated as $\mid C_{50}$ values $\left(\mathrm{ng} \mathrm{ml}^{-1}\right)$

\begin{tabular}{|c|c|c|c|}
\hline Cell line & $I_{50} \mathrm{VCR}$ & $\mathrm{IC}_{50} \mathrm{DOX}$ & $I C_{50}$ CDDP \\
\hline UKF-NB-2 & $0.74 \pm 0.15$ & $8.2 \pm 2.5$ & $|36 \pm 4|$ \\
\hline UKF-NB-2 ${ }^{\mathrm{VCR}}$ & $47.9 \pm 11.2$ & $114.6 \pm 20.7$ & $154 \pm 32$ \\
\hline UKF-NB-2 ${ }^{\text {DOX }}$ & $23.4 \pm 6.4$ & $40.7 \pm 12.3$ & $225 \pm 38$ \\
\hline UKF-NB-2 ${ }^{\text {CDDP }}$ & $1.35 \pm 0.47$ & $17.4 \pm 7.7$ & $819 \pm 52$ \\
\hline UKF-NB-3 & $0.35 \pm 0.09$ & $2.25 \pm 0.78$ & $188 \pm 22$ \\
\hline UKF-NB-3 ${ }^{V C R}$ & $52.7 \pm 9.8$ & $69.1 \pm 15.8$ & $532 \pm 68$ \\
\hline UKF-NB-3 $3^{\mathrm{DOX}}$ & $205 \pm 51.2$ & $62.5 \pm 15.4$ & $231 \pm 22$ \\
\hline UKF-NB-3 $3^{C D D P}$ & $0.84 \pm 0.31$ & $17.3 \pm 2.4$ & $1278 \pm 177$ \\
\hline UKF-NB-6 & $1.91 \pm 0.18$ & $3.5 \pm 0.9$ & $114 \pm 22$ \\
\hline UKF-NB-6VCR & $57.2+11.2$ & $108+12$ & $523+69$ \\
\hline UKF-NB-6 $6^{\text {DOX }}$ & $19.5 \pm 3.4$ & $17.2 \pm 6.5$ & $102 \pm 18$ \\
\hline UKF-NB-6 ${ }^{\text {CDDP }}$ & $3.72 \pm 0.76$ & $21.4 \pm 4.5$ & $2102 \pm 150$ \\
\hline SKNSH & $7.9 \pm 1.3$ & $25.4 \pm 10.1$ & $47.1 \pm 13.5$ \\
\hline
\end{tabular}

Values are from six independent experiments \pm s.d.

Tumour cell adhesion

Adhesion characteristics of UKF-NB-3CDDP

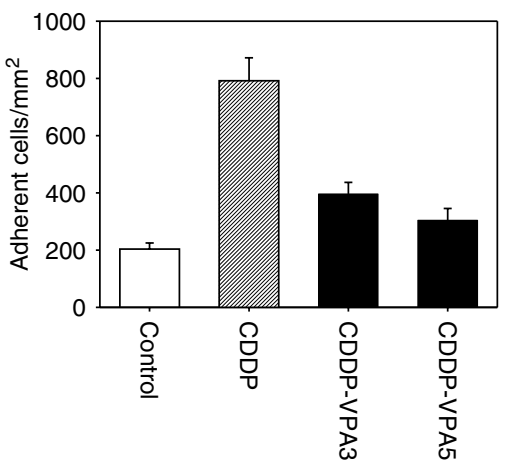

Adhesion characteristics of UKF-NB-3VCR

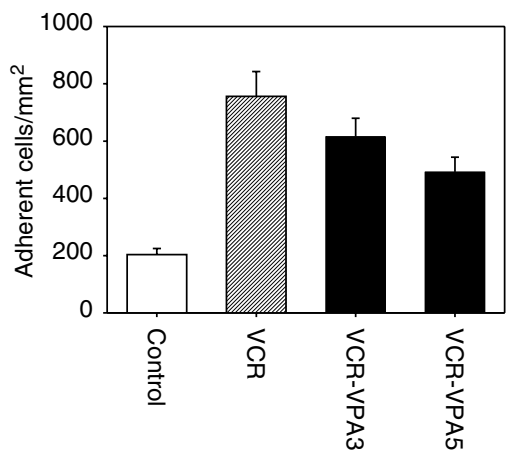

Adhesion characteristics of UKF-NB-3DOX

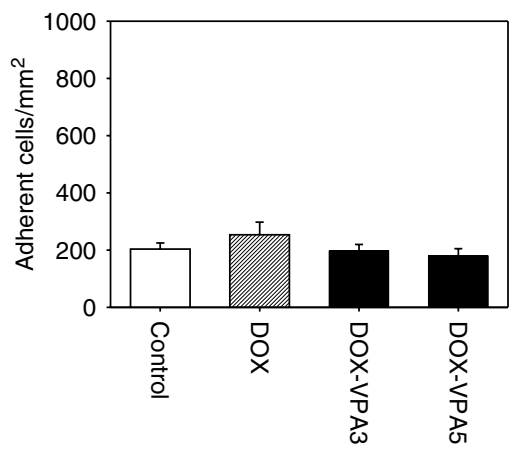

Figure I Valproic acid treatment causes adhesion blockade in CDDP- and VCR-resistant NB cells. The figure depicts adhesion capacity of parental UKFNB-3 (control) vs VCR- (UKF-NB-3 ${ }^{\text {VCR }}$ ). CDDP- (UKF-NB-3 ${ }^{C D D P}$ ) or DOX-resistant NB subpopulations (UKF-NB-3 ${ }^{\text {DOX }}$ ) vs resistant cell lines treated with I mM VPA for 3 (VPA3) or 5 days (VPA5). Neuroblastoma cells were added at a density of $0.5 \times 10^{6}$ cells/well to HUVEC monolayers for 60 min. Nonadherent tumour cells were washed off in each sample, the remaining cells were fixed and counted in five different fields $\left(5 \times 0.25 \mathrm{~mm} \mathrm{~m}^{2}\right)$ using a phase contrast microscope. Adhesion capacity is depicted as tumour cell adhesion $\mathrm{mm}^{-2}$ (mean \pm s.d.; $n=6$ ). 

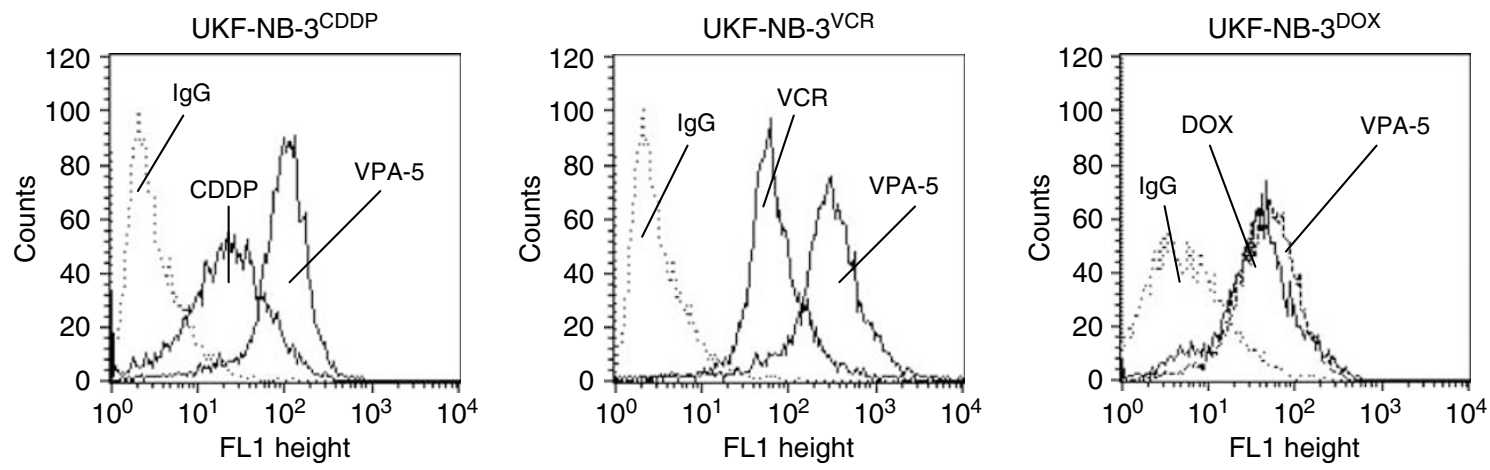

$\begin{array}{ll}\text { IgG isotype control: } & 2.46+/-3.21 \\ \text { Parental control: } & 60.58+/-34.45 \\ \text { Acquired resistance: } & 44.93+/-26.77 \\ \text { Resistance + VPA 3 days: } & 67.85+/-51.29 \\ \text { Resistance + VPA 5 days: } & 123.63+/-66.41\end{array}$

$2.46+/-3.21$
$60.58+/-34.45$
$50.23+/-48.37$
$69.38+/-61.22$
$118.49+/-124.25$

$2.46+/-3.21$

$60.58+/-34.45$

$56.14+/-17.92$

$61.90+/-26.89$

$71.24+/-44.73$

Figure 2 Valproic acid treatment enhances NCAM surface expression in CDDP- and VCR-resistant NB cells. Histograms plots show NCAM surface expression on untreated vs VPA-treated UKF-NB-3 CDDP, UKF-NB-3 ${ }^{\text {VCR }}$ and UKF-NB-3 DOX. IgG isotype controls are also included. The complete experimental data set (MFU \pm s.d.; $n=6$ ) is given below the histograms. Tumour cells were disaggregated mechanically in each experiment and washed in blocking solution. An FITC-conjugated monoclonal antibody anti-CD56, clone 16.2, was used to detect the NCAM I20, I 40 and I $80 \mathrm{kDa}$ isoform. A mouse IgGI-FITC served as the isotype control (IgG). Fluorescence was analysed using a FACScan flow cytometer, and a histogram plot (FLI-Height) was generated to show FITC fluorescence.

Reduction of NCAM surface expression was observed on UKF$\mathrm{NB}-3^{\mathrm{CDDP}}$ and UKF-NB-3 ${ }^{\mathrm{VCR}}$, when compared to the parental control cell line. However, no changes were seen with NCAM expression on UKF-NB-3 ${ }^{\text {DOX }}$, compared to the controls. Treatment of CDDP- or VCR-resistant cell lines with VPA led to a significant increase in NCAM, which exceeded the control values after a 5-day incubation period.

Valproic acid also acted on the parental cell lines, as evidenced by a significant upregulation of NCAM by $+56.9 \pm 22.8 \%(n=5)$.

\section{VPA enhances NCAM protein content}

Similar to the modifications of the NCAM surface expression level, UKF-NB-3 ${ }^{\mathrm{CDDP}}$ and UKF-NB-3 $3^{\mathrm{VCR}}$ were characterised by a strong reduction of NCAM proteins when compared to the parental UKFNB-3 control cells (Figure 3). No differences were seen between UKF-NB-3 and UKF-NB-3 ${ }^{\text {DOX }}$.

When UKF-NB-3 ${ }^{\mathrm{CDDP}}$ or UKF-NB-3 ${ }^{\mathrm{VCR}}$ were treated with VPA for 3 or 5 days, NCAM protein content became upregulated, partially exceeding the control values. Applying VPA to UKF-NB$3^{\text {DOX }}$ did not induce any alterations in NCAM, independent of the incubation time.

\section{VPA modifies NCAM mRNA expression}

Assessment of NCAM mRNA showed distinct expression of mRNA encoding the $140 \mathrm{kDa}$ isoform in UKF-NB-3 control cells, which however became down-modulated in UKF-NB-3 ${ }^{\mathrm{CDDP}}$ or UKF-NB$3^{\mathrm{VCR}}$ (Figure 4). Only slight differences were visualised between UKF-NB-3 and UKF-NB-3 ${ }^{\text {DOX }}$. The presence of VPA was accompanied by elevated $140 \mathrm{kDa}$ mRNA levels in UKF-NB-3 ${ }^{\mathrm{CDDP}}$ or UKF-NB-3 ${ }^{\mathrm{VCR}}$. This effect was not seen in UKF-NB-3 ${ }^{\mathrm{DOX}}$, irrespective if VPA was applied for 3 or 5 days.

Messenger ribonucleic acid encoding the $180 \mathrm{kDa}$ isoforms was not detected in parental UKF-NB-3, nor in UKF-NB-3 ${ }^{\mathrm{CDDP}}$, or UKF$\mathrm{NB}-3^{\mathrm{DOX}}$, and only very weakly expressed in UKF-NB-3 ${ }^{\mathrm{VCR}}$. Surprisingly, VPA evoked NCAM $180 \mathrm{kDa}$ mRNA synthesis already after 3 days in UKF-NB-3 ${ }^{\text {DOX }}$. The same phenomenon was observed after a 5-day VPA treatment in UKF-NB-3 ${ }^{\mathrm{CDDP}}$ or UKF-NB-3 ${ }^{\mathrm{VCR}}$.

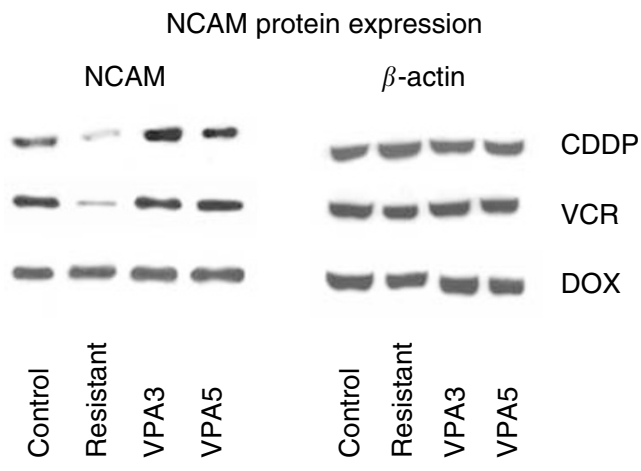

Figure 3 Western blot analysis of NCAM from the proteins of UKF-NB3, UKF-NB-3 $3^{C D D P}$ UKF-NB-3 ${ }^{V C R}$ UKF-NB-3 ${ }^{\mathrm{DOX}}$ and resistant subpopulations treated with VPA for 3 (VPA3) or 5 days (VPA5). Cell lysates were subjected to SDS-PAGE and blotted on the membrane incubated with anti-NCAM (clone 16.2) monoclonal antibodies. $\beta$-Actin served as the internal control. The figure shows one representative from three separate experiments.

\section{VPA alterates p73, deltaNp73 and N-myc expression}

p73, deltaNp73 and N-myc have been identified to trigger NCAM expression (Blaheta et al, 2004). They were therefore used as biomarkers to further explore the influence of VPA on NCAMtriggered NB adhesion. p73 was detected in UKF-NB-3, expression of which was significantly reduced in UKF-NB-3 ${ }^{\text {CDDP }}$ and UKF$\mathrm{NB}-3^{\mathrm{VCR}}$. Doxorubicin resistance was not accompanied by a distinct p73 downregulation (Table 2). When VPA was added to the cell cultures, downregulation of p73 seen in UKF-NB-3 $3^{\mathrm{CDDP}}$ and UKF-NB- $3^{\mathrm{VCR}}$ was reverted and the protein became upregulated after 3 and 5 days. deltaNp73 became enhanced in UKF-NB$3^{\mathrm{CDDP}}$ and UKF-NB-3 ${ }^{\mathrm{VCR}}$ compared to the drug naïve UKF-NB-3 controls. Application of VPA led to a significant reduction of deltaNp73 in CDDP- or VCR-resistant cell lines.

$\mathrm{N}$-myc proteins were enhanced in UKF-NB-3 ${ }^{\mathrm{CDDP}}$ and UKF-NB$3^{\mathrm{VCR}}$, but not in UKF-NB-3 $3^{\mathrm{DOX}}$, compared to the UKF-NB-3 control cell line (Figure 5). Moderate N-myc downregulation was induced by 
VPA in UKF-NB-3 ${ }^{\mathrm{VCR}}$. N-myc became nearly undetectable in UKFNB-3 ${ }^{\text {CDDP }}$ after a 5-day treatment with VPA. The distinct influence of $\mathrm{VPA}$ on UKF-NB-3 ${ }^{\mathrm{CDDP}}$ or UKF-NB-3 ${ }^{\mathrm{VCR}}$ was also proven by flow cytometry, which revealed left shifting of the $\mathrm{N}$-myc-specific fluorescence intensity, indicating $\mathrm{N}$-myc loss. $\mathrm{N}$-myc histogram

\section{NCAM mRNA analysis}

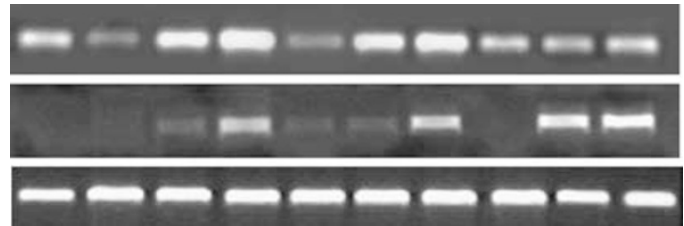

NCAM140

NCAM180

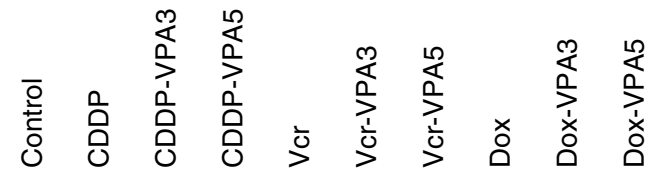

Figure 4 RT-PCR analysis of NCAM I40 and I $80 \mathrm{kDa}$ RNA in UKFNB-3, UKF-NB-3 CDDP UKF-NB-3 $3^{V C R}$ UKF-NB-3 ${ }^{\mathrm{DOX}}$ and resistant subpopulations treated with VPA for 3 (VPA3) or 5 days (VPA5). Ribonucleic acid were extracted, reverse-transcribed and submitted to semiquantitative RT-PCR using gene-specific primers as indicated in Materials and Methods. The internal control for the RT-PCR reaction was performed by running parallel reaction mixtures with the housekeeping gene GAPDH. The figure shows one representative from three separate experiments. analysis concentrates on isotype controls and specific fluorescence of untreated $v s$ VPA-treated (5-day treatment) UKF-NB-3 ${ }^{\mathrm{CDDP}}$, UKFNB-3 ${ }^{\mathrm{VCR}}$ and UKF-NB-3 ${ }^{\mathrm{DOX}}$. The complete experimental data set (MFU \pm s.d.; $n=6$ ) is given below the histogram.

\section{VPA acts on further NB tumour cell lines}

To strengthen the relevance of our findings, further NB tumour cell lines were included in the study. Analysis of UKF-NB-2 and

Table 2 Mean fluorescence units showing influence of VPA on p73 and deltaNp73 expression in drug-resistant neuroblastoma cells

\begin{tabular}{lcc}
\hline Cell line & p73 & deltaNp73 \\
\hline UKF-NB-3 & $18.29 \pm 6.25$ & $11.51 \pm 8.34$ \\
CDDP & $3.38 \pm 3.64^{\mathrm{a}}$ & $19.71 \pm 7.68^{\mathrm{a}}$ \\
CDDP+VPA3 & $8.16 \pm 5.66^{\mathrm{b}}$ & $12.44 \pm 6.94^{\mathrm{b}}$ \\
CDDP+VPA5 & $12.8 \pm 7.32^{\mathrm{b}}$ & $11.06 \pm 6.72^{\mathrm{b}}$ \\
VCR & $5.23 \pm 4.78^{\mathrm{a}}$ & $26.17 \pm 13.95^{\mathrm{a}}$ \\
VCR+VPA3 & $13.78 \pm 6.13^{\mathrm{b}}$ & $17.33 \pm 8.99^{\mathrm{b}}$ \\
VCR+VPA5 & $17.62 \pm 8.72^{\mathrm{b}}$ & $11.23 \pm 6.85^{\mathrm{b}}$ \\
DOX & $15.46 \pm 8.29$ & $10.02 \pm 4.29$ \\
DOX+VPA3 & $17.90 \pm 11.21$ & $10.64 \pm 6.78$ \\
DOX+VPA5 & $19.95 \pm 13.96$ & $11.95 \pm 3.81$
\end{tabular}

Values are from six independent experiments \pm s.d. CDDP indicates UKF-NB-3 ${ }^{C D D P}$, VCR indicates UKF-NB-3 ${ }^{\text {VCR }}$, DOX indicates UKF-NB-3 ${ }^{\text {DOX. }}$. VPA was added for 3 (VPA3) or 5 days (VPA5). ${ }^{a}$ Indicates significant difference to UKF-NB-3. 'Indicates significant difference to the drug-resistant NB sublines.

$\mathrm{N}$-myc expression level

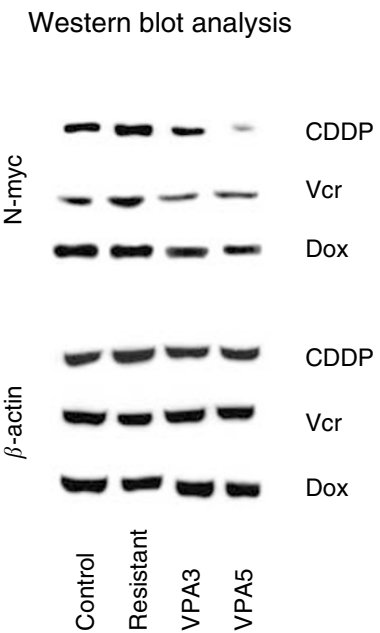

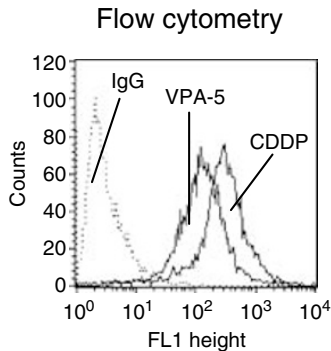
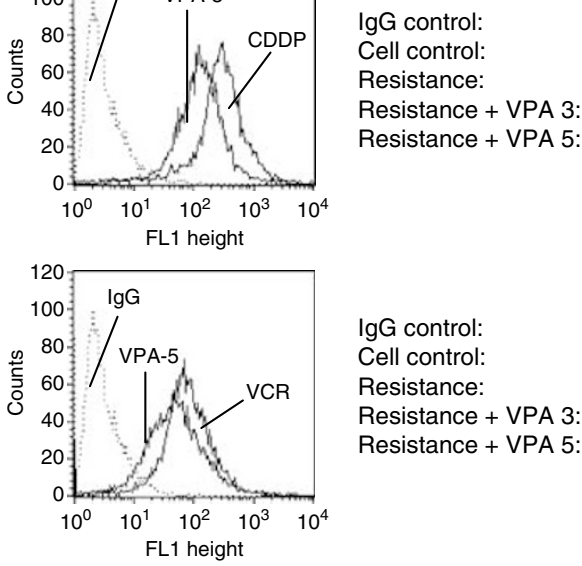

IgG control:

Cell control:

$3.69+/-2.54$

Resistance:

$82.36+/-57.22$

$109.72+/-78.38$

Resistance + VPA 3: $99.76+/-56.91$

Resistance + VPA 5: $71.72+/-42.47$

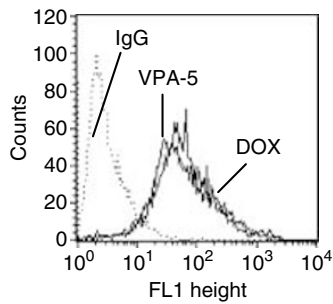

IgG control: $\quad 3.69+/-2.54$

Cell control: $\quad 82.36+/-57.22$

Resistance: $\quad 84.08+/-66.45$

Resistance + VPA 3: $86.35+/-72.21$

Resistance + VPA 5: $81.07+/-36.43$

Figure 5 Valproic acid alters N-myc expression in CDDP- and VCR-resistant UKF-NB-3 cells. The right part of the figure depicts results from flow cytometry analysis. To allow intracellular staining, tumour cells were fixed and permeabilised by methanol-acetone before antibodies were added. To identify N-myc, the monoclonal anti-N-myc antibody clone NCM || I00 was used. Primary antibodies were labelled with goat anti-mouse IgG-FITC. Background staining was evaluated by goat anti-mouse IgG-FITC. The histograms plots show N-myc expression level in untreated vs VPA-treated UKF-NB$3^{C D B P}$, UKF-NB-3 ${ }^{\mathrm{VCR}}$ and UKF-NB-3 ${ }^{\mathrm{DOX}}$. IgG isotype controls are also included. The complete experimental data set $(M F U+\mathrm{s} . \mathrm{d}$.; $n=6)$ is given alongside. The left part of the figure shows Western blot analysis of N-myc from the proteins of UKF-NB-3, UKF-NB-3 $3^{\mathrm{CDDP}}$ UKF-NB-3 $3^{\mathrm{CR}}$ UKF-NB-3 ${ }^{\mathrm{D} B \times}$ and resistant subpopulations treated with VPA for 3 (VPA3) or 5 days (VPA5). Cell lysates were subjected to SDS-PAGE and blotted on the membrane incubated with anti-N-myc monoclonal antibodies. $\beta$-Actin served as the internal control. The figure shows one representative from three separate experiments. 
Table 3 Comparative analysis of adhesion capacity, NCAM and N-myc expression level of several neuroblastoma cells and drug-resistant sublines

\begin{tabular}{|c|c|c|c|}
\hline Cell line & $\begin{array}{l}\text { Cell adhesion } \\
\text { to HUVEC } \\
\left(\text { cells } \mathrm{mm}^{-2}\right)\end{array}$ & $\begin{array}{l}\text { NCAM } \\
\text { expression } \\
\text { (MFU) }\end{array}$ & $\begin{array}{c}N \text {-myc } \\
\text { expression } \\
\text { (MFU) }\end{array}$ \\
\hline UKF-NB-2 & 75.2 & 188.2 & 40.1 \\
\hline UKF-NB-2 \pm VPA & $60.8^{c}$ & $212.6^{c}$ & $28.6^{\mathrm{c}}$ \\
\hline UKF-NB-2 ${ }^{\overline{C D D P}}$ & $318.4^{\mathrm{a}}$ & $82.8^{a}$ & 39.6 \\
\hline UKF-NB-2 ${ }^{C D D P} \pm V P A$ & $175.2^{b}$ & $168.9^{b}$ & $32.2^{\mathrm{b}}$ \\
\hline UKF-NB-2 $2^{V C R}$ & $245.6^{a}$ & $99.7^{\mathrm{a}}$ & 44.7 \\
\hline UKF-NB-2 $2^{V C R} \pm V P A$ & $121.6^{\mathrm{b}}$ & $154.6^{\mathrm{b}}$ & 42.3 \\
\hline UKF-NB-2 ${ }^{\text {DOX }}$ & $172.6^{\mathrm{a}}$ & $127.9^{\mathrm{a}}$ & 38.8 \\
\hline UKF-NB- $2^{\mathrm{DOX}} \pm \mathrm{VPA}$ & 124.5 & 143.5 & 39.4 \\
\hline UKF-NB-6 & 72.4 & 256.8 & 339.3 \\
\hline UKF-NB-6 + VPA & $41.0^{c}$ & $316.8^{c}$ & $238.0^{c}$ \\
\hline UKF-NB-6 CDDP & 816 & 2487 & 305.8 \\
\hline UKF-NB-6 $6^{\mathrm{CDDP}} \pm \mathrm{VPA}$ & $56.8^{\mathrm{b}}$ & $283.8^{b}$ & $224.2^{b}$ \\
\hline UKF-NB-6 ${ }^{V C R}$ & $115.6^{\mathrm{a}}$ & $181.0^{\mathrm{a}}$ & 298.9 \\
\hline UKF-NB-6 $6^{\mathrm{VCR}} \pm \mathrm{VPA}$ & $79.2^{b}$ & $284.2^{b}$ & 331.6 \\
\hline UKF-NB-6 $6^{\text {DOX }}$ & 73.8 & 251.3 & 344.0 \\
\hline UKF-NB- $6^{\mathrm{DOX}} \pm$ VPA & 71.9 & 254.5 & 321.7 \\
\hline SKNSH & 56.8 & 318.9 & 17.7 \\
\hline SKNSH \pm VPA & $27.2^{c}$ & $812.1^{c}$ & 18.6 \\
\hline
\end{tabular}

$M F U=$ mean fluorescence units. ${ }^{a}$ Indicates significant difference to the parental neuroblastoma cell line. ${ }^{b}$ Indicates significant difference to the drug-resistant tumour sublines. ${ }^{~ I n d i c a t e s ~ s i g n i f i c a n t ~ d i f f e r e n c e ~ b e t w e e n ~ V P A-t r e a t e d ~ p a r e n t a l ~ c e l l s ~ a n d ~ t h e i r ~}$ non-treated controls. Mean standard deviations were as follows: cell adhesion intra-assay $<25 \%$, cell adhesion inter-assay $<80 \%$. NCAM expression intra-assay $<5 \%$, NCAM expression $_{\text {inter-assay }}<30 \%$. N-myc expression intra-assay $_{2}<5, \quad \mathrm{~N}$-myc expression $_{\text {inter-assay }}<50 \%$.

drug-resistant sublines indicated a significant increase in UKF-NB$2^{\mathrm{CDDP}}, \mathrm{UKF}-\mathrm{NB}-2^{\mathrm{VCR}}$ and UKF-NB-2 $2^{\mathrm{DOX}}$ cell adhesion, compared to the parental controls. Adhesion correlated inversely with the NCAM surface level (Table 3). A similar phenomenon was observed in CDDP- (moderate) and VCR-resistant UKF-NB-6 cell lines. A 3-day VPA treatment blocked cell binding to HUVEC in UKF-NB-2 and their drug-resistant sublines, and upregulated NCAM expression. Valproic acid also acted on UKF-NB-6, UKFNB- $6^{\text {CDDP }}$, UKF-NB- $6^{\text {VCR }}$ and SKNSH. However, although VPA (partially) reverted the increased adhesion phenotype and restored NCAM levels, it did not re-sensitise the drug-resistant NB cells to VCR, DOX or CDDP (data not shown).

$\mathrm{N}$-myc was significantly diminished by VPA in UKF-NB-2, UKFNB-2 ${ }^{\mathrm{CDDP}}$, UKF-NB-6 and UKF-NB-6 ${ }^{\mathrm{CDDP}}$, compared to the controls. However, no differences were seen between VPA-treated and non-treated VCR or DOX-resistant tumour cell lines.

To better interprete our data, antiproliferative effects of VPA on the chemosensitive $v s$ chemoresistant NB cells were analysed in final experiments. Table 4 document growth inhibition by this compound, whereas higher VPA concentrations were necessary to reach 50\% reduction in chemotherapy-resistant cell lines compared to the parental controls.

\section{DISCUSSION}

Based on this cell culture model, we have demonstrated that drugresistant NB cancer cells develop an increased malignant phenotype as evidenced by enhanced adhesion to vascular endothelial cells, accompanied by significant downregulation of the adhesion receptor NCAM. Valproic acid reverted this process by downregulating cell adhesion and upregulating NCAM expression. Considering clinical utility, it is encouraging that VPA was active in NB cell lines resistant to existing chemotherapies, since overcoming resistance to anticancer agents is a major challenge in the development of novel antitumour protocols.
Table 4 Antiproliferative effects of VPA, indicated as $\mathrm{IC}_{50}$ values (mM)

\begin{tabular}{lc}
\hline Cell line & IC $_{\mathbf{5 0}}$ \\
\hline UKF-NB-2 & $0.44 \pm 0.31$ \\
UKF-NB-2 CDDP & $1.85 \pm 0.58$ \\
UKF-NB-2 VCR & $1.36 \pm 0.62$ \\
UKF-NB-2 & $1.44 \pm 0.42$ \\
UKF-NB-3 & $1.03 \pm 0.37$ \\
UKF-NB-3 & $1.51 \pm 0.44$ \\
UKF-NB-3 $\mathrm{VCR}$ & $1.88 \pm 0.57$ \\
UKF-NB-3 & $2.18 \pm 0.76$ \\
UKF-NB-6 & $1.27 \pm 0.53$ \\
UKF-NB-6 & $1.53 \pm 0.46$ \\
UKF-NB-6 & $2.36 \pm 0.71$ \\
UKF-NB & $0.93 \pm 0.39$ \\
SKNSH-6 & $3.16 \pm 0.78$ \\
\hline
\end{tabular}

Values are from six independent experiments \pm s.d.

In fact, several cases have been documented showing antineoplastic effects of VPA in patients with relapsed tumours. When VPA was given as maintenance therapy for childhood malignant glioma after postoperative combined chemotherapy and irradiation, about $10 \%$ of these patients were maintained in continuous complete remission and an equal number of patients showed at least partial responses (Blaheta et al, 2005). An additional pediatric patient with glioblastoma multiforme responded to VPA after showing progressive disease shortly after having received combined chemotherapy and irradiation as well as topotecane (Witt et al, 2004). Another pediatric patient suffering from a relapsed supratentorial primitive neuroectodermal tumour while receiving chemotherapy (CCNU, VCR and cisplatinum) after total resection and irradiation showed conspicuous signs of glial differentiation induction and a nonmalignant morphology on histological examination. This patient had received VPA for epilepsy treatment for a period of several months before the tumour recurred (Driever et al, 2004).

In vitro, VPA has been shown to inhibit proliferation in acute myeloid leukaemia cells expressing P-glycoprotein (P-gp) and MDR-associated protein 1 (Tang et al, 2004), and to increase sensitivity towards apoptosis in hepatoma cells resistant to epirubicin (Schuchmann et al, 2006). Although the underlying mode of action has not been explored in these studies, the data clearly indicate that VPA may alter the malignant behaviour of tumours that do not respond to chemotherapy. With particular emphasis on NB, VPA significantly prevented the interaction between tumour cells and endothelium. This finding is important, because binding of single cancer cells to the vessel wall represents the first step in the haematogenous invasion cascade proceeding transendothelial migration and invasion into surrounding tissue. We therefore conclude that VPA may have a direct impact on metastasis formation. In good accordance to this hypothesis, VPA enhanced the NCAM surface level, expression of which is strongly involved in tumour cell adhesion and penetration.

In primitive neuroectodermal tumour cells, an increase in NCAM was paralleled by a significant reduction in cellular motility and adhesion capacity (Owens et al, 1998; Prag et al, 2002). In a rat model, NCAM-transfected glioma tumour cells became less invasive and destructive than control cells with a low NCAM expression level (Edvardsen et al, 1994). Diminished expression of NCAM was also associated with clinically aggressive colon cancers (Sampson-Johannes et al, 1996; Roesler et al, 1997; Huerta et al, 2001), and dissemination of pancreatic $\beta$-tumour cells (Perl et al, 1999; Cavallaro et al, 2001). Tezel et al (2001) suggested that NCAM expression in tubular adenocarcinoma of the pancreas has a significant impact on overall patient survival. We recently demonstrated an inverse correlation between NCAM expression and NB cell adhesion, assessed on $11 \mathrm{NB}$ cell lines. In particular, transfection with a cDNA encoding the human NCAM-140 kD 
isoform enhanced NCAM expression and diminished initial NB cell adhesion, treatment with NCAM antisense oligonucleotides reduced NCAM surface level and induced upregulation of NB cell adhesion to endothelium (Blaheta et al, 2002). It is currently assumed that NCAM, in its function as a homophilic receptor, stabilises the primary tumour or tumour cell aggregates, while circulating in the blood vessels. Reduction of the NCAM expression level might lead to a reduction in cell-cell binding forces, and hence to the release of tumours as single cells. The less NCAM, the more metastatic cells leave the tumour mass, and the more penetration events can take place (Blaheta et al, 2002). Consequently, NCAM upregulation observed in CDDP- and VCRresistant $\mathrm{NB}$ tumour cells under VPA might reduce cell transmigration and extravasation processes.

There is some evidence from the literature that N-myc downregulates NCAM expression, thus increasing the invasiveness of NB cells. Transfection of the rat NB cell line B104 with an N-myc expression vector resulted in a dramatic reduction in the levels of NCAM polypeptides and mRNAs, and increased metastatic ability (Akeson and Bernards, 1990). Cytomegalovirus-induced acceleration of NB adhesion and transendothelial penetration was evoked by increasing $\mathrm{N}$-myc protein content, accompanied by a diminished NCAM surface level (Blaheta et al, 2004). The present data reveal strong upregulation of N-myc in UKF-NB-3 ${ }^{\mathrm{CDDP}}$, compared to the parental cells, process of which was coupled to NCAM loss and enhanced adhesion capacity. It may therefore be concluded that $\mathrm{N}$-myc plays an important role in NCAM-driven NB adhesion, and that VPA has an impact on $\mathrm{N}$-myc protein expression. Nevertheless, the situation is more complex than initially thought. Our analysis on further NB cell lines indicated that VPA reverts cell adhesion, restored NCAM and suppressed N-myc expression level on UKF-NB-2, UKF-NB-6 and their CDDP-resistant sublines. However, VPA effects on VCR-resistant sublines were not accompanied by $\mathrm{N}$-myc alterations. Furthermore, incubation of multidrug-resistant SKNSH with VPA induced very strong adhesion blockade and NCAM upregulation, although N-myc was detected in non-treated controls just very slightly over threshold values. Based on this, we assume that endogenous $\mathrm{N}$ myc expression level of NB cells may not correlate with their responsiveness to VPA-induced NCAM upregulation, and VPAinduced loss of $\mathrm{N}$-myc may be limited to CDDP-resistant and drug-sensitive tumour cells.

p73 and deltaNp73, an isoform of p73 lacking the N-terminal transactivation domain, are both suggested to be associated with NCAM expression. In vitro experiments demonstrated that transfected full-length p73 cDNA induces expression of NCAM and downregulation of $\mathrm{N}-\mathrm{myc}$ in $\mathrm{N} 1 \mathrm{E}-115 \mathrm{NB}$ cells. Inversely, transfection of dominant-negative p73 abrogated the transactivation of the NCAM promoter (De Laurenzi et al, 2000). Based on UKF-NB-4 tumour cells, we have recently postulated a direct association between tumour progression, upregulation of N-myc and deltaNp73, and downregulation of p73 and NCAM (Blaheta et al, 2004). According to this statement, VPA evoked UKF-NB-3 adhesion blockade was accompanied by diminished $\mathrm{N}$-myc and deltaNp73, and an enhanced p73 and NCAM level. With special respect to the UKF-NB-3 model, VPA may interfere in the N-myc/ deltaNp73 signalling system that causes, as at least one consequence, distinct upregulation of NCAM biosynthesis and receptor processing. Neural cell adhesion molecule processing finally attributes to the lowered invasive capacity of the tumour cells.
To summarise, evidence is presented showing that drugresistant NB cells are sensitive to VPA treatment. Valproic acid distinctly reduced the invasive properties of $\mathrm{NB}$ and may therefore be well suited to amend the current treatment protocol with particular emphasis on those tumours that do not respond to chemotherapy. Nevertheless, our data are particularly limited to four NB cell lines. Therefore, the hypothetical possibility that VPA might overcome drug resistance in general needs further investigation. Remarkably, VPA blocked cell adhesion of parental, but not of DOX-resistant UKF-NB-3 or UKF-NB-6 tumour cells in our assay. This implies that DOX may, under certain circumstances, induce resistance to VPA. A similar phenomenon was found using NB cells with 'naturally' arised DOX resistance, SMS-KANR and SMS-KCNR (Reynolds et al, 1986). Tabe et al (2006) demonstrated in this context that the HDAC-inhibitor FK228 induces P-gp expression and prevents growth inhibition and apoptosis in acute promyelocytic leukaemia cells subsequently incubated with DOX. Okada et al (2006) observed resistance development in DOX-resistant clones of osteosarcoma and Ewing's family of tumours after exposure to FK228. Nevertheless, FK228 is chemically different from VPA and, therefore, further experiments are necessary to explore this issue.

Differences between VCR/CDDP- and DOX-resistant cell lines may also point to different resistance mechanisms that are operational in these cell lines. Suppression of MAP kinase (MEKERK) signalling has been observed in NB cells with acquired resistance to DOX (Mattingly et al, 2001; Armstrong et al, 2006). Down-modulation of ERK1/2 phosphorylation has further been documented in NB cells with acquired resistance to CDDP or VCR. However, these cell lines were additionally characterised by a distinct Akt activation (Kotchetkov et al, 2005; Servidei et al, 2006). Therefore, although purely speculative, fine-tuned alterations of the ERK and Akt signalling system may be - at least partially - responsible for establishing VCR and CDDP, but not DOX resistance in our NB cell model. However, detailed knowledge of resistance mechanism in individual cancer cells is necessary to allow better prediction of the clinical use of VPA.

Other HDAC inhibitors have also been shown to inhibit tumour growth and to overcome multidrug resistance. Notably, suberoylanilide hydroxamic acid (SAHA) has been demonstrated to act on VCR-resistant leukaemia cell lines (Ruefli et al, 2002), adriamycinresistant breast (Castro-Galache et al, 2003) and paclitaxel-resistant ovarian cancer cells (Sonnemann et al, 2006). SAHA at $5 \mu \mathrm{M}$ significantly diminished UKF-NB-3 ${ }^{\mathrm{CDDP}}$ or UKF-NB-3 ${ }^{\mathrm{VCR}}$ proliferation and cell adhesion to HUVEC in our own experiments (data not shown). Histone deacetylase inhibitors different from VPA may therefore be considered to become additional options for the treatment of drug-resistant NB. However, detailed studies are necessary to explore their clinical value.

\section{ACKNOWLEDGEMENTS}

We thank Karen Nelson for critically reading the manuscript. This work was supported by the foundation 'Hilfe für krebskranke Kinder Frankfurt e. V', the 'Heinrich und Erna Schaufler-Stiftung', the 'Horst Müggenburg-Stiftung', the 'Matthias Lackas-Stiftung' and the 'Jung-Stiftung'.

\section{REFERENCES}

Akeson R, Bernards R (1990) N-myc down regulates neural cell adhesion molecule expression in rat neuroblastoma. Mol Cell Biol 10: 2012-2016 Armstrong MB, Bian X, Liu Y, Subramanian C, Ratanaproeksa AB, Shao F, Yu VC, Kwok RP, Opipari Jr AW, Castle VP (2006) Signaling from p53 to
NF-kappaB determines the chemotherapy responsiveness of neuroblastoma. Neoplasia 8: $967-977$

Blaheta RA, Kronenberger B, Woitaschek D, Weber S, Scholz M, Schuldes H, Encke A, Markus BH (1998) Development of an ultrasensitive in vitro 
assay to monitor growth of primary cell cultures with reduced mitotic activity. J Immunol Methods 211: 159-169

Blaheta RA, Hundemer M, Mayer G, Vogel JU, Kornhuber B, Cinatl J, Markus BH, Driever PH, Cinatl Jr J (2002) Expression level of neural cell adhesion molecule (NCAM) inversely correlates with the ability of neuroblastoma cells to adhere to endothelium in vitro. Cell Commun Adhes 9: $131-147$

Blaheta RA, Beecken WD, Engl T, Jonas D, Oppermann E, Hundemer M, Doerr HW, Scholz M, Cinatl J (2004) Human cytomegalovirus infection of tumor cells downregulates NCAM (CD56): a novel mechanism for virus-induced tumor invasiveness. Neoplasia 6: 323-331

Blaheta RA, Michaelis M, Driever PH, Cinatl Jr J (2005) Evolving anticancer drug valproic acid: insights into the mechanism and clinical studies. Med Res Rev 25: $383-397$

Castro-Galache MD, Ferragut JA, Barbera VM, Martin-Orozco E, GonzalezRos JM, Garcia-Morales P, Saceda M (2003) Susceptibility of multidrug resistance tumor cells to apoptosis induction by histone deacetylase inhibitors. Int J Cancer 104: 579-586

Cavallaro U, Niedermeyer J, Fuxa M, Christofori G (2001) N-CAM modulates tumour-cell adhesion to matrix by inducing FGF-receptor signalling. Nat Cell Biol 3: 650-657

Cinatl Jr J, Cinatl J, Scholz M, Driever PH, Henrich D, Kabickova H, Vogel JU, Doerr HW, Kornhuber B (1996) Antitumor activity of sodium valproate in cultures of human neuroblastoma cells. Anticancer Drugs 7: $766-773$

De Laurenzi V, Raschella G, Barcaroli D, Annicchiarico-Petruzzelli M, Ranalli M, Catani MV, Tanno B, Costanzo A, Levrero M, Melino G (2000) Induction of neuronal differentiation by $\mathrm{p} 73$ in a neuroblastoma cell line. J Biol Chem 275: 15226-15231

Driever PH, Wagner S, Hofstadter F, Wolff JE (2004) Valproic acid induces differentiation of a supratentorial primitive neuroectodermal tumor. Pediatr Hematol Oncol 21: 743-751

Edvardsen K, Pedersen P-H, Bjerkvig R, Hermann GG, Zeuthen J, Laerum OD, Walsh FS, Bock E (1994) Transfection of glioma cells with the neural-cell adhesion molecule NCAM: effect on glioma cell invasion and growth in vivo. Int $J$ Cancer 58: 116-122

Hirokawa Y, Arnold M, Nakajima H, Zalcberg J, Maruta H (2005) Signal therapy of breast cancers by the HDAC inhibitor FK228 that blocks the activation of PAK1 and abrogates the tamoxifen-resistance. Cancer Biol Ther 4: $956-960$

Huerta S, Srivatsan ES, Venkatesan N, Peters J, Moatamed F, Renner S, Livingston EH (2001) Alternative mRNA splicing in colon cancer causes loss of expression of neural cell adhesion molecule. Surgery 130: 834-843

Keshelava N, Seeger RC, Groshen S, Reynolds CP (1998) Drug resistance patterns of human neuroblastoma cell lines derived from patients at different phases of therapy. Cancer Res 58: 5396-5405

Kleinschmidt-DeMasters BK, Orr EA, Savelieva E, Owens GC, Kruse CA (1999) Paucity of retinoic acid receptor alpha (RAR alpha) nuclear immunostaining in gliomas and inability of retinoic acid to influence neural cell adhesion molecule (NCAM) expression. J Neurooncol 41: 31-42

Kotchetkov R, Cinatl J, Blaheta R, Vogel JU, Karaskova J, Squire J, Hernaiz Driever P, Klingebiel T, Cinatl Jr J (2003) Development of resistance to vincristine and doxorubicin in neuroblastoma alters malignant properties and induces additional karyotype changes: a preclinical model. Int I Cancer 104: 36-43

Kotchetkov R, Driever PH, Cinatl J, Michaelis M, Karaskova J, Blaheta R, Squire JA, Von Deimling A, Moog J, Cinatl Jr J (2005) Increased malignant behavior in neuroblastoma cells with acquired multi-drug resistance does not depend on P-gp expression. Int J Oncol 27: 1029-1037

Maiso P, Carvajal-Vergara X, Ocio EM, Lopez-Perez R, Mateo G, Gutierrez N, Atadja P, Pandiella A, San Miguel JF (2006) The histone deacetylase inhibitor LBH589 is a potent antimyeloma agent that overcomes drug resistance. Cancer Res 66: 5781-5789

Mattingly RR, Milstein ML, Mirkin BL (2001) Down-regulation of growth factor-stimulated MAP kinase signaling in cytotoxic drug-resistant human neuroblastoma cells. Cell Signal 13: 499-505
Okada T, Tanaka K, Nakatani F, Sakimura R, Matsunobu T, Li X, Hanada M, Nakamura T, Oda Y, Tsuneyoshi M, Iwamoto Y (2006) Involvement of P-glycoprotein and MRP1 in resistance to cyclic tetrapeptide subfamily of histone deacetylase inhibitors in the drug-resistant osteosarcoma and Ewing's sarcoma cells. Int J Cancer 118: 90 - 97

Owens GC, Orr EA, DeMasters BK, Muschel RJ, Berens ME, Kruse CA (1998) Overexpression of a transmembrane isoform of neural cell adhesion molecule alters the invasiveness of rat CNS-1 glioma. Cancer Res 58: $2020-2028$

Pathil A, Armeanu S, Venturelli S, Mascagni P, Weiss TS, Gregor M, Lauer UM, Bitzer M (2006) HDAC inhibitor treatment of hepatoma cells induces both TRAIL-independent apoptosis and restoration of sensitivity to TRAIL. Hepatology 43: 425-434

Perl AK, Dahl U, Wilgenbus P, Cremer H, Semb H, Christofori G (1999) Reduced expression of neural cell adhesion molecule induces metastatic dissemination of pancreatic beta tumor cells. Nat Med 5: 286-291

Prag S, Lepekhin EA, Kolkova K, Hartmann-Petersen R, Kawa A, Walmod PS, Belman V, Gallagher HC, Berezin V, Bock E, Pedersen N (2002) NCAM regulates cell motility. J Cell Sci 115: 283-292

Reynolds CP, Biedler JL, Spengler BA, Reynolds DA, Ross RA, Frenkel EP, Smith RG (1986) Characterization of human neuroblastoma cell lines established before and after therapy. J Natl Cancer Inst 76: 375-387

Roesler J, Srivatsan E, Moatamed F, Peters J, Livingston EH (1997) Tumor suppressor activity of neural cell adhesion molecule in colon carcinoma. Am J Surg 174: $251-257$

Ruefli AA, Bernhard D, Tainton KM, Kofler R, Smyth MJ, Johnstone RW (2002) Suberoylanilide hydroxamic acid (SAHA) overcomes multidrug resistance and induces cell death in P-glycoprotein-expressing cells. Int $J$ Cancer 99: $292-298$

Sampson-Johannes A, Wang W, Shtivelman E (1996) Colonization of human lung grafts in SCID-hu mice by human colon carcinoma cells. Int J Cancer 65: $864-869$

Schuchmann M, Schulze-Bergkamen H, Fleischer B, Schattenberg JM, Siebler J, Weinmann A, Teufel A, Worns M, Fischer T, Strand S, Lohse AW, Galle PR (2006) Histone deacetylase inhibition by valproic acid down-regulates c-FLIP/CASH and sensitizes hepatoma cells towards CD95- and TRAIL receptor-mediated apoptosis and chemotherapy. Oncol Rep 15: $227-230$

Servidei T, Riccardi A, Sanguinetti M, Dominici C, Riccardi R (2006) Increased sensitivity to the platelet-derived growth factor (PDGF) receptor inhibitor STI571 in chemoresistant glioma cells is associated with enhanced PDGF-BB-mediated signaling and STI571-induced Akt inactivation. J Cell Physiol 208: $220-228$

Sonnemann J, Gange J, Pilz S, Stotzer C, Ohlinger R, Belau A, Lorenz G, Beck JF (2006) Comparative evaluation of the treatment efficacy of suberoylanilide hydroxamic acid (SAHA) and paclitaxel in ovarian cancer cell lines and primary ovarian cancer cells from patients. $B M C$ Cancer 6: 183

Tabe Y, Konopleva M, Contractor R, Munsell M, Schober WD, Jin L, Tsutsumi-Ishii Y, Nagaoka I, Igari J, Andreeff M (2006) Up-regulation of MDR1 and induction of doxorubicin resistance by histone deacetylase inhibitor depsipeptide (FK228) and ATRA in acute promyelocytic leukemia cells. Blood 107: 1546-1554

Tang R, Faussat AM, Majdak P, Perrot JY, Chaoui D, Legrand O, Marie JP (2004) Valproic acid inhibits proliferation and induces apoptosis in acute myeloid leukemia cells expressing P-gp and MRP1. Leukemia 18: $1246-1251$

Tezel E, Kawase Y, Takeda S, Oshima K, Nakao A (2001) Expression of neural cell adhesion molecule in pancreatic cancer. Pancreas 22: 122-125

Tsurutani J, Soda H, Oka M, Suenaga M, Doi S, Nakamura Y, Nakatomi K, Shiozawa K, Amada YY, Kamihira S, Kohno S (2003) Antiproliferative effects of the histone deacetylase inhibitor FR901228 on small-cell lung cancer lines and drug-resistant sublines. Int J Cancer 104: 238-242

Witt O, Schweigerer L, Driever PH, Wolff J, Pekrun A (2004) Valproic acid treatment of glioblastoma multiforme in a child. Pediatr Blood Cancer 43: 181 